\title{
The in vivo reduction of afferent facilitation induced by low frequency electrical stimulation of the motor cortex is antagonized by cathodal direct current stimulation of the cerebellum
}

\author{
Nordeyn Oulad Ben Taib ${ }^{1}$ and Mario Manto $2,3^{*}$
}

\begin{abstract}
Background: Low-frequency electrical stimulation to the motor cortex (LFSMC) depresses the excitability of motor circuits by long-term depression (LTD)-like effects. The interactions between LFSMC and cathodal direct current stimulation (CDCS) over the cerebellum are unknown.

Methods: We assessed the corticomotor responses and the afferent facilitation of corticomotor responses during a conditioning paradigm in anaesthetized rats. We applied LFSMC at a frequency of $1 \mathrm{~Hz}$ and a combination of LFSMC with CDCS.

Results: LFSMC significantly depressed both the corticomotor responses and the afferent facilitation of corticomotor responses. Simultaneous application of cDCS over the cerebellum antagonized the depression of corticomotor responses and cancelled the depression of the afferent facilitation.

Conclusion: Our results demonstrate that CDCS of the cerebellum is a potent modulator the inhibition of the motor circuits induced by LFSMC applied in vivo. These results expand our understanding of the effects of cerebellar DCS on motor commands and open novel applications for a cerebellar remote control of LFSMC-induced neuroplasticity. We suggest that the cerebellum acts as a neuronal machine supervising not only long-term potentiation (LTP)-like effects, but also LTD-like effects in the motor cortex, two mechanisms which underlie cerebello-cerebral interactions and the cerebellar control of remote plasticity. Implications for clinical ataxiology are discussed.
\end{abstract}

Keywords: Neuromodulation, Plasticity, Motor cortex, Cerebellum, Direct current stimulation, In vivo

\section{Background}

Direct current stimulation (DCS) is growingly applied to understand the roles of the cerebellum on the sensorimotor or cognitive operations, and to modulate the effects of the cerebellum over the cerebral cortex in ataxic disorders [1]. Anodal DCS (aDCS) of the cerebellum reinforces the inhibition exerted by the Purkinje neurons

\footnotetext{
* Correspondence: mmanto@ulb.ac.be

¿Unité d'Etude du Mouvement (UEM), FNRS, Neurologie ULB-Erasme, 808 Route de Lennik, 1070 Bruxelles, Belgium

${ }^{3}$ Service des Neurosciences, Université de Mons, 7000 Mons, Belgium

Full list of author information is available at the end of the article
}

over cerebellar nuclei, whereas cathodal DCS (cDCS) of the cerebellum induces a disinhibition of cerebellar nuclei, which physiologically excite thalamic targets amongst others. In other words, aDCS decreases the excitatory drive exerted by cerebellar nuclei, with opposite effects of cDCS [2]. The polarity-specific modulation of cerebellar-motor cortex connectivity is currently mainly explained by changes of the activity of the cerebellothalamo-cortical pathways [2]. These tracts represent the best candidates for gating of the information flow from the cerebellum to the cerebral cortex [3]. Cerebellar nuclei project in particular to thalamic nuclei which target 
themselves the layers IV and V of the primary motor cortex, whose horizontal neuronal connections represent a substrate for map reorganization during plasticity [4].

It has been demonstrated that low frequency stimulation of the motor cortex $(\leq 1 \mathrm{~Hz})$ with repeated TMS (rTMS) exerts powerful inhibitory effects on corticospinal excitability by synaptic mechanisms similar to long-term depression (LTD) [5]. These effects are site-specific, unaffecting the contralateral motor cortex. The increased inhibition of the motor cortex might participate in the deficits observed in cerebellar disorders [6,7]. Since cDCS of the cerebellum disinhibits cerebellar nuclei, this technique might be useful to antagonize the decreased excitability of the motor cortex in cerebellar patients. We tested the hypothesis that cDCS of the cerebellum counteracts the inhibitory effects exerted by low frequency electrical stimulation of the motor cortex (LFSMC).

\section{Methods}

Experiments were approved by the Animal Care Committee of ULB. We made all efforts to reduce animal suffering as much as possible and to use the minimal number of animals. Adult Wistar rats $(n=11$; weight between 240 and $390 \mathrm{~g})$ were anaesthetized with chloral hydrate $(400 \mathrm{mg} / \mathrm{kg}$ i.p., followed by a continuous infusion to obtain a steady-state anesthesia after about 15 min and reproducible motor evoked potentials MEPs; CMA micropump, CMA, Sweden) before the beginning of the surgical procedure [8]. Rats were put in a stereotaxic apparatus (Kaps, Germany). Scalp was shaved and cut sagitally. The tissue overlying the cranium was removed (epicranial stimulation to obtain corticomotor responses and epidural stimulation for CDCS; see below). Body temperature was maintained between 36.0 and $37.5^{\circ} \mathrm{C}$. Indeed, this parameter is critical for the activity of glutamatergic pathways [9].

\section{Experimental protocol}

The following protocol was applied:
A. Baseline Measurements (MEPs and conditioned corticomotor responses)
B. LFSMC (T0-T10 min)
C. Measurements post-LFSMC (T20 min)
D. Measurements post-LFSMC (T45 $\mathrm{min})$
E. $\mathrm{cDCS}+\mathrm{LFSMC}$ (T50-T60 min)
F. Measurements post-cDCS/LFSMC (T70 min)

\section{Motor threshold (MT) and Motor evoked potentials (MEPs)}

We first determined the "hot spot" of the left gastrocnemius muscle by stimulating the right motor cortex using a mapping procedure (matrix of $6 \times 9$ sites) [10]. Stimulation was applied every $\mathrm{mm}$ in the sagittal axis and every $0.5 \mathrm{~mm}$ in the coronal axis (epicranial stimulation). We used a successive point-by-point stimulation method with monophasic pulses. The duration of electrical stimuli (square waves) was $1 \mathrm{msec}$ (NeuroMax 4, Xltek, Canada). The right motor cortex was stimulated at an intensity of $130 \%$ of the motor threshold MT (defined as the minimal intensity eliciting at least 5 out of 10 evoked responses with an amplitude $>20 \mu \mathrm{V})$. We measured peak-to-peak amplitudes of MEPs (sets of 10 corticomotor responses were considered to compute the mean responses). We inserted subcutaneous needle electrodes (Technomed 017K25) in left gastrocnemius muscle to record MEPs. Impedance was maintained below $5 \mathrm{KOhms.}$

\section{Conditioned corticomotor responses}

The conditioning stimulus (DS70 stimulator, Digitimer, UK) was delivered in the left sciatic nerve (stimulation at a distance of about $16 \mathrm{~mm}$ laterally from midline; intensity of stimulation eliciting a small twitch of the hindlimb) at an inter-stimulus interval (ISI) of $6 \mathrm{msec}$ before application of a test stimulus on right motor cortex. Such short ISIs are associated with an afferent facilitation whereas long ISIs are associated with an afferent inhibition [10].

\section{Low-frequency electrical stimulation to the motor cortex (LFSMC)}

For LFSMC, we administered squared pulses (duration: $1 \mathrm{msec}$ ) at an intensity corresponding to the MT at a frequency of $1 \mathrm{~Hz}$ during $10 \mathrm{~min}$ (600 stimuli) over the right motor cortex after the following baseline measurements: (1) a set of 10 MEPs without conditioning, followed by (2) a set of 10 duos of MEPs for the conditioning paradigm [10]. LFSMC was applied from T0 min to T10 min (current supplied by a constant current stimulator A310-A365, World Precision Instruments, UK). rTMS at the frequency of $1 \mathrm{~Hz}$ and at an intensity corresponding to $\mathrm{MT}$ is known to elicit a strong reduction in motor cortex excitability [5]. Durations of 5 to 20 min have been applied in rats by other groups using rTMS [5]. We first observed (in 3 rats) that the depression of MEPs was maximal from T10 to T30 min. The depression lasted about 25 min (amplitudes of MEPs returned to baseline values at about T35 $\mathrm{min})$. We repeated the measurements of MEPs (10 MEPs without conditioning and 10 duos of MEPs in the conditioning paradigm) $10 \mathrm{~min}$ after the end of application of LFSMC (T20 min). We selected to combine cDCS (see next section) with LFSMC $50 \mathrm{~min}$ (from T50 to T60 min) after the beginning of LFSMC, when the excitability of the motor cortex had returned to baseline values. 


\section{Cathodal transcranial direct current stimulation (CDCS) over the cerebellum}

cDCS was applied over left cerebellar hemisphere during 10 min in conjunction with the application of LFSMC applied over the right motor cortex (from T50 to T60 min). The method to apply cDCS has been reported earlier [11]. This is based on the study of Fregni et al. [12]. The anode (low impedance metallic electrode with a diameter of $0.8 \mathrm{~mm}$ ) was fixed $5 \mathrm{~mm}$ anterior to the bregma in right supraorbital region, inserted epicranially. A small plastic jacket was fixed over left cerebellar hemisphere with dental cement and filled with saline solution $(0.9 \% \mathrm{NaCl})$ to obtain a contact area of $7.1 \mathrm{~mm}^{2}$. The cathode was applied epidurally over the left cerebellar hemisphere. cDCS was applied directly onto the dura to ensure a defined contact area over the cerebellar cortex. The after-effects of cDCS last about 55-65 min [11]. We previously showed that cDCS does not change the amplitudes of MEPs, redistributes corticomotor maps and does not modify the afferent inhibition [11]. Measurements of MEPs (10 MEPs without conditioning and 10 duos) were repeated at T70 min. At the end of the experiments, an overdose of chloral hydrate $(1000 \mathrm{mg} /$ kg i.p.) was administered. Following decapitation, brains were extracted and examined under a microscope to exclude local lesions or bleeding.

\section{Statistical analysis}

Statistical analysis was performed using Sigma Stat (Jandel Scientific, Germany). The normality of data was assessed using the Kolmogorov-Smirnov test. We compared the amplitudes of MEPs without conditioning before LFSMC (baseline; T0 min), after LFSMC (T20 min), at T45 min (to confirm return to baseline values' range) and after the combination cDCS/LFSMC (at T70 min) using the Friedman repeated measures analysis of variance on ranks, followed by pairwise multiple comparison procedures with the Tukey test. We compared the conditioned responses (afferent facilitation: ratio of conditioned response CR by unconditioned response UR) in the 4 recording times (at T0, T20, T45, T70 min) using the repeated measures analysis of variance, followed by the Tukey test. Despite the results of the normality assessment for conditioned responses, we also computed a Friedman repeated measures analysis of variance on ranks followed by the Tukey test given our sample size. Statistical significance was set at $p=0.05$.

\section{Results}

We did not observe lesions induced by electrical stimulation or bleeding in the motor cortex or in the cerebellum. The amplitudes of MEPs were significantly depressed by LFSMC. However, this LFSMC-induced depression was antagonized by cDCS of the cerebellum. This is illustrated in Fig. 1 (top panels). The afferent facilitation (assessed by the ratios of CR divided by UR) was depressed by LFSMC (Fig. 1, bottom panels). cDSC of the cerebellum antagonized the effects of LFSMC and even unbalanced the effects of LFSMC.

For the amplitudes of corticomotor responses, normality test failed $(p<0.05)$. The Friedman test showed a statistically significant difference between the 4 conditions (Fig. 2a; $p<0.001$, coefficient of concordance of 0.763 and average rank $r$ of 0.739 ). Tukey test showed that amplitudes of MEPs were significantly smaller at T20 min as compared to baseline (T0 $\mathrm{min}$ ), T45 min and T70 min $(p<0.05)$. Normality test passed for the conditioned corticomotor responses in the paradigm of afferent facilitation ( $p=0.119$; equal variance test: $p=0.115$ ). The analysis of variance showed a statistically significant difference between the 4 conditions (Fig. 2b; $F=78,92$ with $p<0.001$, coefficient of concordance of 0.878 and average rank $\mathrm{r}$ of 0.866 ). Post-hoc multiple comparisons revealed that ratios of CR divided by UR were significantly smaller at T20 min as compared to baseline, T45 min and T70 min $(p<0.001)$. cDCS entirely reverted the effects of LFSMC and even surpassed these effects. Indeed, ratios were significantly greater at T70 $\mathrm{min}$ as compared to T0 $\mathrm{min}(p=0.034)$, and at T70 $\mathrm{min}$ as compared to T45 $\min (p=0.003)$. There was no statistical difference between $\mathrm{T} 0 \mathrm{~min}$ and $\mathrm{T} 45 \mathrm{~min}(p=0.751)$, confirming that the excitability of the motor cortex had returned to the range of baseline values. ${ }^{1}$

\section{Discussion}

We provide the first experimental evidence that $\mathrm{cDCS}$ of the cerebellum antagonizes the strong inhibitory effect exerted by LFSMC applied over the contralateral motor cortex, expanding our understanding of the numerous and complex interactions between motor cortex and cerebellum [13]. We focused on the gastrocnemius muscle and cannot extrapolate the results to other limb muscles.

At this stage, we cannot distinguish between (a) an antagonistic effect of $\mathrm{cDCS}$ on the plasticity related changes induced by LFSMC, (b) an additive modulation of corticomotoneuronal output, and (c) a combination of the two mechanisms. Further studies are required, including single-cell recordings in the various layers of the cerebral cortex, especially recordings of inhibitory interneurons and pyramidal cells in the deep cortical layers. In addition, an effect upon extra-pyramidal pathways and/or spinal cord cannot be ruled out. Both the ratios Mean F/Mean $M$ response and the persistence of $F$ waves are significantly increased in the ipsilateral gastrocnemius muscle after application of cDCS of the cerebellum alone [11]. By contrast, cDCS does not modify the amplitudes of $\mathrm{H}$ reflex. We have demonstrated in a 


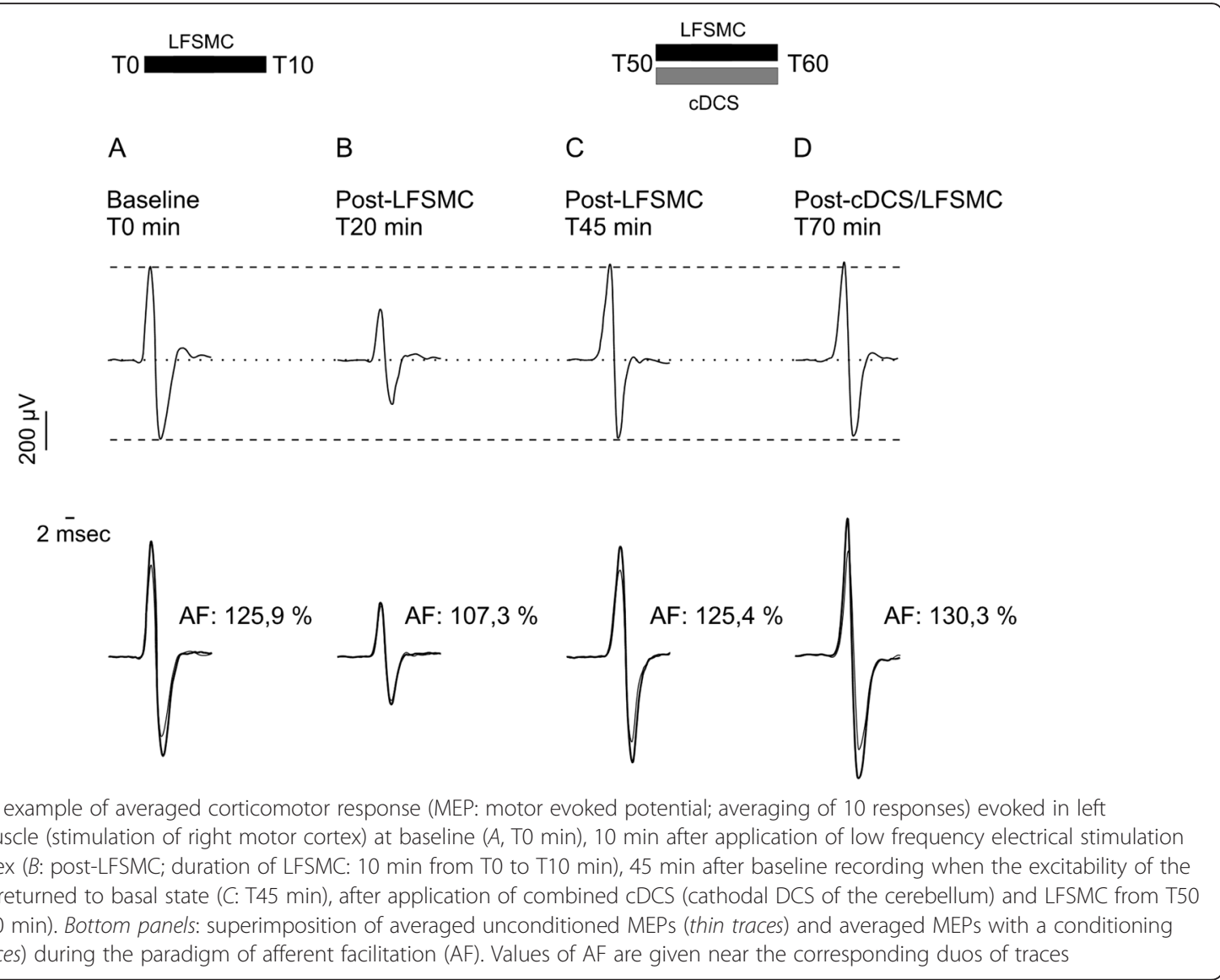

previous study that LFRSMC at an intensity of $130 \%$ of MT (frequency of $1 \mathrm{~Hz}$ ) changes the inter-hemispheric inhibition (IHI) but does not modify the excitability of the anterior horn motoneurons pool [14]. Therefore, repetitive stimulation of $\mathrm{M} 1$ at $1 \mathrm{~Hz}$ at an intensity of MT is unlikely to change the excitability of the spinal cord. In rats, pyramidal neurons and GABAergic interneurons of deep cortical layers receive directly the interhemispheric information [15]. Pyramidal neurons of layer VI respond monosynaptically to callosal stimulation [16]. This argues for a direct effect of low frequency stimulation upon the cerebral cortex itself. Layer VI pyramidal cells have wide projections towards other cortical areas [16]. Callosal information contributes to a bilateral corticothalamic integration by modulating the activity of inhibitory interneurons involved in cellular plasticity [15]. The interhemispheric inhibition (IHI) is a keymechanism for the balance of activities between the 2 hemispheres [14]. Because the anode was fixed anterior to the bregma in right supraorbital region for the cDCS, a contribution of callosal pathways should be considered in our experiment. Moreover, the study of Fregni et al. on cortical spreading depression (a wave of neuronal depolarization propagating across the cortical surface) in rats argues for a direct effect of repetitive electrical stimulation at $1 \mathrm{~Hz}$ upon the cerebral cortex [12].

One limitation of the study is the use of continuous anaesthesia, a factor which might impact on the mechanisms of plasticity of the brain and which might influence our results. In our model, continuous infusion of chloral hydrate is required to obtain reproducible corticomotor responses. Although the half-life of chloral hydrate is short (a few minutes), the half-lives of the metabolites (trichloroethanol TCE and trichloroacetic acid TCA) are longer (up to $60 \mathrm{~h}$ ) [17]. The mechanisms of action of chloral hydrate remain poorly understood but are known to involve GABAergic pathways, which are implicated in brain excitability and plasticity, especially for long-term plasticity [18]. Ideally, brain plasticity should be investigated without administration of anesthetic agents. This remains a major challenge for the experiments on corticomotor responses in vivo in rodents.

The excitability of the motor cortex can be tuned by acting directly on the motor cortex such as applying LFSMC, or by acting on anatomical structures targetting the motor cortex such as the prefrontal cortex, the sensory cortex or thalamic nuclei. For instance, the motor commands from M1 can be shaped by a modulation of the activity of rFr2 (prefrontal area, the equivalent of the 


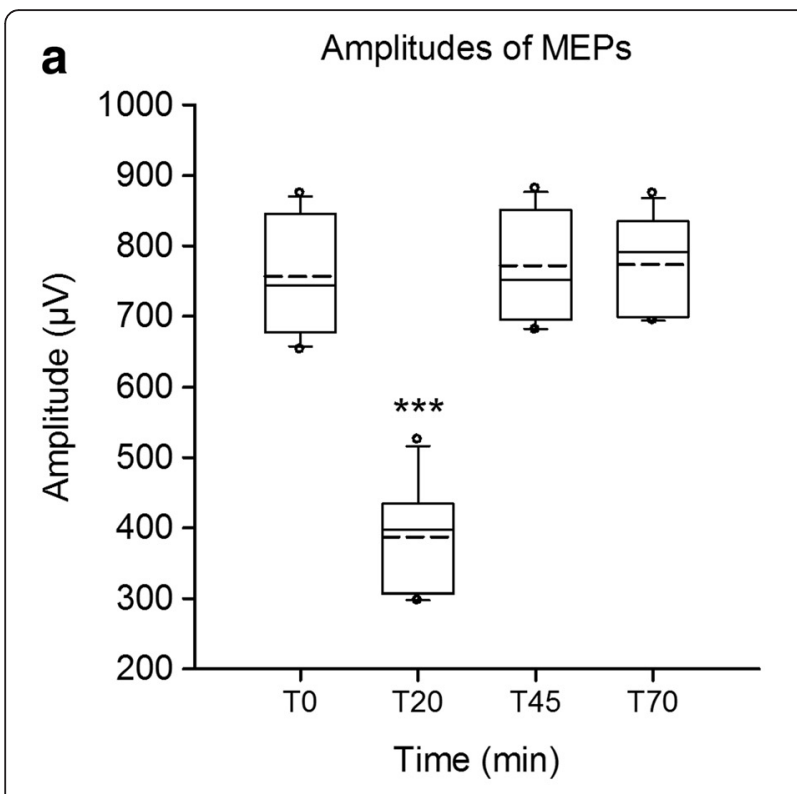

\section{b Afferent Facilitation (Conditioned MEPs)}

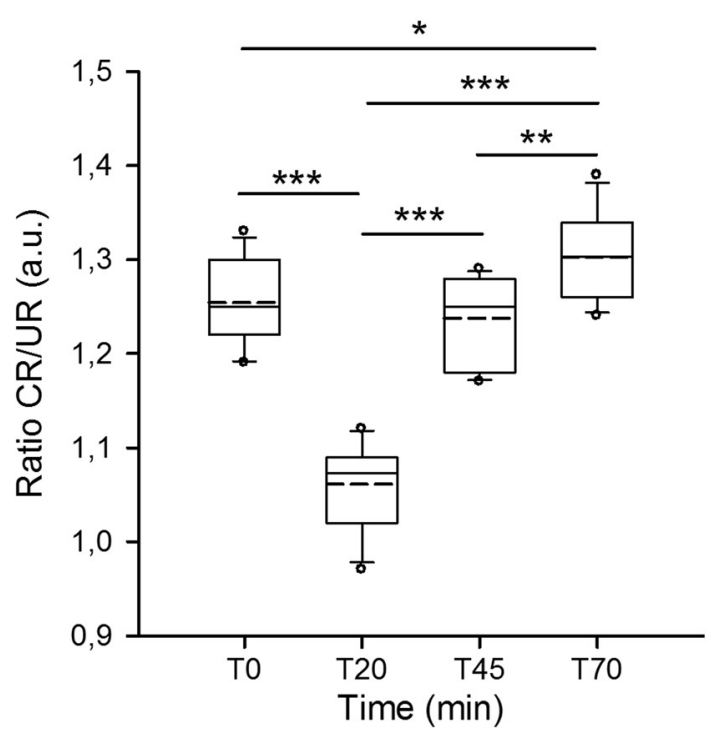

Fig. 2 a: box and whisker plots of amplitudes of MEPs expressed in $\mu \mathrm{V} .{ }^{* * *}: p<0.001$ as compared to T0, T45 and T70 min. b: box and whisker plots of afferent facilitation. Ratios of conditioned (CR)/ unconditioned (UR) are shown at T0, T20, T45 and T70 min. Ratios are expressed in arbitrary units (a.u.). Medians (continuous lines), dotted lines (mean values) and outliers are illustrated. *: $p<0.05$, $*^{* *}: p<0.01,{ }^{* * *}: p<0.001$

premotor/supplementary motor areas in primates) [17-20]. The mechanism of afferent facilitation is enhanced if preceded by trains of electrical stimulation applied over rFr2 [10]. It has also been shown that repetitive somatosensory peripheral stimulation increases the excitability of the motor cortex and that an intact cerebellum is required for this form of short-term brain plasticity [21]. The activity of thalamic nuclei can be modified by acting on the cerebellar circuitry [22, 23]. The cerebello-dentatothalamo-cortical pathway is a major actor in the anatomofunctional dialogue between the motor cortex and the cerebellum. The cerebellum is particularly responsive to electrical stimulation for anatomical and physiological reasons [24-26]. Modelling studies of cerebellar DCS indicate that the electric field (E) and the current density (J) spatial distributions occur mainly in the cerebellar cortex, with negligible spreads towards the brainstem [25]. Studies on cerebellar cortex ablation have confirmed the importance of Purkinje cell firings upon the discharges of cerebellar nuclei [27]. Such lesions cause a considerable increase in the background firing and cancel the pauses in discharges occurring in responses induced by somatosensory stimuli.

We have discussed previously the main anatomical pathways involved in the modulation of corticomotoneuronal output and the effects of rTMS on the excitability of the motor cortex [10]. Whereas transcranial electrical stimulation excites directly the axons of pyramidal neurons and generate direct (D) waves, TMS evokes several volleys of corticospinal activity : D-waves from direct axonal activation and later waves (I-waves) resulting from activation of mono- and polysynaptic inputs to pyramidal neurons $[28,29]$. TMS excites the pyramidal neurons transsynaptically [29]. With both transcranial electrical stimulation and TMS, high-frequency synchronized descending volleys of activity are recorded in the epidural space [30-32]. However, there is still some debate on the synaptic mechanisms at the origin of I waves [33].

aDCS and cDCS of the cerebellum cannot just be considered as having pure opposite effects. Using an ISI of $45 \mathrm{msec}, \mathrm{aDCS}$ of the cerebellum enhances the afferent inhibition of conditioned corticomotor responses, unlike cDCS which has no significant impact on the afferent inhibition [11]. aDCS decreases the amplitude of corticomotor responses and changes the representation pattern of limb muscles over the motor cortex. A "focusing effect" is observed, with a concentration of the highest motor responses around the hot spot. In this case, an opposite effect occurs with cDCS.

MEP suppression by $1 \mathrm{~Hz}$ rTMS has been demonstrated in rats under general anesthesia [5]. Several authors consider that repetitive electrical stimulation of the cerebral cortex in the rat mimics the effects of magnetic stimulation [12]. A long-term depression (LTD)type plasticity is suggested to explain MEP suppression. Indeed, the effects of rTMS are known (1) to be frequency-dependent, (2) to outlast the period of stimulation, and (3) to rely on NMDA pathways [5, 34, 35]. Our results show that the neuromodulation of cerebellar activity by cDCS exerts powerful remote effects on the LTD-like plasticity induced by LFSMC. Therefore, the 2 techniques appear to compete in terms of consequences 
on motor cortex excitability. Regarding rTMS, its direct application over the cerebellum tunes the activity of contralateral motor cortex. One Hz rTMS over the cerebellar cortex increases intracortical facilitation (ICF) at the level of contralateral M1, and low-frequency cerebellar rTMS trains affect motor intracortical excitability beyond the application of the train [36]. ICF is depressed in hemicerebellectomized rats but remains responsive to trains of stimulations applied over the prefrontal cortex [10].

What are the implications in the field of clinical ataxiology? We propose the following potential therapeutical applications of DCS in cerebellar patients, still deserving further confirmations in specific clinical studies. Lesions or dysfunction of cerebellar nuclei induce a depression of contralateral motor cortex excitability which can be reverted either by aDCS of the motor cortex $[37,38]$ or by cDCS of the cerebellum which disinhibits cerebellar nuclei. Examples of dysfunction of cerebellar nuclei are compression by tumors of the surrounding white matter or hydrocephalus, intoxications [37, 39], spinocerebellar ataxia type 3 (SCA3) or Friedreich ataxia which are associated with a nuclear grumose degeneration [40, 41], calcium deposits $[42,43]$. It is currently unclear whether the recently reported deposits of gadolinium in cerebellar nuclei of patients having received multiple administrations will be associated with very slowly evolving cerebellar deficits (motor, cognitive and/or affective) or will remain clinically silent for life [44]. Lesions of the cerebellar cortex (cerebellitis, cerebellar cortical atrophy) lead to a disinhibition of cerebellar nuclei and overactivity of contralateral motor cortex. Application of LFSMC or aDCS of the cerebellum could be administered to regulate motor output. It was shown recently that aDCS improves ataxias associated with cerebellar atrophy [45]. Disorders combining a pathology of the cerebellum and hyperexcitability of the motor cortex could benefit from combinations of LFSMC and DCS of the cerebellum. One example is familial cortical myoclonic tremor with epilepsy [46, 47]. Finally, the modulation of the motor cortex excitability is considered as an early change before structural plasticity $[48,49]$ and therefore combinations of LFSMC/DCS of the cerebellum may find future applications in the attempts to influence sensorimotor learning with a clinical perspective in mind. On a broader perspective, the remote supervision of LTD-like mechanisms in the cerebral cortex might be a mean to act on sensori-motor learning disorders and could be envisioned as a novel tool to appreciate the functional preservation of the cerebellar projections towards the primary motor cortex, complementing the previously reported properties of DCS in the detection of very early lesions in the cerebral cortex [50] and adding another electrophysiological tool to assess cerebellum-brain interactions [51]. The hypothesis that the cerebellum filters or processes time-specific incoming sensory volleys in order to influence the plasticity of the motor cortex is reinforced by findings of impaired long-term potentiation (LTP)-like effects during paired associative stimulation [52]. Overall, cerebellum appears as key-actor for the supervision of remote plasticity in the motor cortex.

\section{Conclusion}

This is the first demonstration that cDCS of the cerebellum modulates the effects of LFSMC upon the excitability of motor circuits. Our results potentially open future applications for a cerebellar remote control of LFSMCinduced neuroplasticity in vivo.

\section{Endnotes}

${ }^{1}$ Given the sample size, a Friedman repeated measures analysis of variance on ranks was also applied despite the values of the normality assessment. The $p$ value (the 4 conditions being considered) was $<0.001$. Tukey test showed that values at T70 min were greater than values at T45 min and T20 min, and that values at T0 min were greater than values at T20 min. However, values at $\mathrm{T} 20 \mathrm{~min}$ are similar to values at $\mathrm{T} 45 \mathrm{~min}$. In addition, values at $\mathrm{T} 70 \mathrm{~min}$ were similar to values at $\mathrm{T} 0 \mathrm{~min}$.

\section{Acknowledgments}

MM is supported by the FNRS-Belgium.

\section{Authors' contributions}

NOBT and MM conceived the study, performed the experiments and drafted the manuscript. Both the authors have read and approved the final version of the manuscript.

\section{Competing interests}

The authors declare that they have no competing interests.

\section{Author details}

'Department of Neurosurgery, CHU Saint-Pierre, Rue Haute, 1000 Bruxelles, Belgium. ${ }^{2}$ Unité d'Etude du Mouvement (UEM), FNRS, Neurologie ULB-Erasme, 808 Route de Lennik, 1070 Bruxelles, Belgium. ${ }^{3}$ Service des Neurosciences, Université de Mons, 7000 Mons, Belgium.

Received: 1 April 2016 Accepted: 25 August 2016

Published online: 30 August 2016

\section{References}

1. Grimaldi G, Argyropoulos GP, Boehringer A, Celnik P, Edwards MJ, Ferrucci R, Galea JM, Groiss SJ, Hiraoka K, Kassavetis P, Lesage E, Manto M, Miall RC, Priori A, Sadnicka A, Ugawa Y, Ziemann U. Non-invasive cerebellar stimulation-a consensus paper. Cerebellum. 2014;13(1):121-38.

2. Grimaldi G, Argyropoulos GP, Bastian A, Cortes M, Davis NJ, Edwards DJ, Ferrucci R, Fregni F, Galea JM, Hamada M, Manto M, Miall RC, Morales-Quezada L, Pope PA, Priori A, Rothwell J, Tomlinson SP, Celnik P. Cerebellar transcranial direct current stimulation (ctDCS): a novel approach to understanding cerebellar function in health and disease. Neuroscientist. 2016;22(1):83-97.

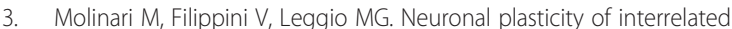
cerebellar and cortical networks. Neuroscience. 2002;111(4):863-70.

4. Sanes JN, Donoghue JP. Plasticity and primary motor cortex. Annu Rev Neurosci. 2000;23:393-415.

5. Muller PA, Dhamne SC, Vahabzadeh-Hagh AM, Pascual-Leone A, Jensen FE, Rotenberg A. Suppression of motor cortical excitability in anesthetized rats 
by low frequency repetitive transcranial magnetic stimulation. PLoS One. 2014;9(3):e91065.

6. Di Lazzaro V, Restuccia D, Nardone R, Leggio MG, Oliviero A, Profice P, Tonali P, Molinari M. Motor cortex changes in a patient with hemicerebellectomy. Electroencephalogr Clin Neurophysiol. 1995;97(5):259-63.

7. Farias da Guarda SN, Cohen LG, da Cunha Pinho M, Yamamoto FI, Marchiori PE, Scaff M, Conforto AB. Interhemispheric asymmetry of corticomotor excitability after chronic cerebellar infarcts. Cerebellum. 2010;9(3):398-404.

8. Manto M, Dalmau J, Didelot A, Rogemond V, Honnorat J. Afferent facilitation of corticomotor responses is increased by lgGs of patients with NMDA-receptor antibodies. J Neurol. 2011;258:27-33.

9. Cais O, Sedlacek M, Horak M, Dittert I, Vyklicky Jr L. Temperature dependence of NR1/NR2B NMDA receptor channels. Neuroscience. 2008;151(2):428-38.

10. Oulad Ben Taib N, Manto M. Effects of trains of high-frequency stimulation of the premotor/supplementary motor area on conditioned corticomotor responses in hemicerebellectomized rats. Exp Neurol. 2008;212(1):157-65.

11. Oulad Ben Taib N, Manto M. Trains of epidural DC stimulation of the cerebellum tune corticomotor excitability. Neural Plast. 2013;2013:613197.

12. Fregni F, Liebetanz D, Monte-Silva KK, Oliveira MB, Santos AA, Nitsche MA, Pascual-Leone A, Guedes RC. Effects of transcranial direct current stimulation coupled with repetitive electrical stimulation on cortical spreading depression. Exp Neurol. 2007;204(1):462-6.

13. Manto M. On the cerebello-cerebral interactions. Cerebellum. 2006;5(4):286-8.

14. Oulad Ben Taib N, Manto M. Reinstating the ability of the motor cortex to modulate cutaneomuscular reflexes in hemicerebellectomized rats. Brain Res. 2008;1204:59-68.

15. Karayannis T, Huerta-Ocampo I, Capogna M. GABAergic and pyramidal neurons of deep cortical layers directly receive and differently integrate callosal input. Cereb Cortex. 2007;17(5):1213-26.

16. Zhang ZW, Deschênes M. Projections to layer VI of the posteromedial barrel field in the rat: a reappraisal of the role of corticothalamic pathways. Cereb Cortex. 1998;8(5):428-36.

17. Gauillard J, Cheref S, Vacherontrystram MN, Martin JC. Chloral hydrate: a hypnotic best forgotten? Encéphale. 2002;28(3 Pt 1):200-4.

18. Bachtiar V, Stagg CJ. The role of inhibition in human motor cortical plasticity. Neuroscience. 2014;278:93-104.

19. Hicks RR, Huerta MF. Differential thalamic connectivity of rostral and caudal parts of cortical area Fr2 in rats. Brain Res. 1991;568(1-2):325-9.

20. Takase K, Mitsushima D, Funabashi T, Kimura F. Sex difference in the 24-h acetylcholine release profile in the premotor/supplementary motor area of behaving rats. Brain Res. 2007;1154:105-15.

21. Farias da Guarda F, Conforto AB. Effects of somatosensory stimulation on corticomotor excitability in patients with unilateral cerebellar infarcts and healthy subjects - preliminary results. Cerebellum Ataxias. 2014;1:16.

22. Ando N, Izawa $Y$, Shinoda $Y$. Relative contributions of thalamic reticular nucleus neurons and intrinsic interneurons to inhibition of thalamic neurons projecting to the motor cortex. J Neurophysiol. 1995;73(6):2470-85.

23. Pinault $D$, Deschênes $M$. The origin of rhythmic fast subthreshold depolarizations in thalamic relay cells of rats under urethane anaesthesia. Brain Res. 1992:595(2):295-300.

24. Lu MK, Tsai CH, Ziemann U. Cerebellum to motor cortex paired associative stimulation induces bidirectional STDP-like plasticity in human motor cortex. Front Hum Neurosci. 2012;6:260.

25. Parazzini M, Rossi E, Ferrucci R, Liorni I, Priori A, Ravazzani P. Modelling the electric field and the current density generated by cerebellar transcranial DC stimulation in humans. Clin Neurophysiol. 2014;125(3):577-84.

26. Ferrucci R, Cortese F, Priori A. Cerebellar tDCS: how to do it. Cerebellum. 2015;14(1):27-30.

27. Tarnecki R. Responses of the red nucleus neurons to limb stimulation after cerebellar lesions. Cerebellum. 2003;2(2):96-100.

28. Volz LJ, Hamada M, Rothwell JC, Grefkes C. What makes the muscle twitch: motor system connectivity and TMS-induced activity. Cereb Cortex. 2015;25(9):2346-53.

29. Terao Y, Ugawa Y. Basic mechanisms of TMS. J Clin Neurophysiol. 2002;19(4):322-43.

30. Boyd S, Rothwell J, Cowan J, et al. A method of monitoring note on motor conduction velocities. J Neurol Neurosurg Psychiatry. 1986;49:251e7.
31. Nakamura H, Kitagawa H, Kawaguchi Y, Tsuji H. Intracortical facilitation and inhibition after transcranial magnetic stimulation in conscious humans. J Physiol Lond. 1997;498:817e23.

32. Di Lazzaro V, Oliviero A, Saturno E, et al. The effect on corticospinal volleys of reversing the direction of current induced in the motor cortex by transcranial magnetic stimulation. Exp Brain Res. 2001;138:268e73.

33. Rusu CV, Murakami M, Ziemann U, Triesch J. A model of TMS-induced I-waves in motor cortex. Brain Stimul. 2014;7(3):401-14.

34. Kobayashi M. Effect of slow repetitive TMS of the motor cortex on ipsilateral sequential simple finger movements and motor skill learning. Restor Neurol Neurosci. 2010;28:437-48.

35. Padberg F, George MS. Repetitive transcranial magnetic stimulation of the prefrontal cortex in depression. Exp Neurol. 2009;219:2-13.

36. Fierro B, Giglia G, Palermo A, Pecoraro C, Scalia S, Brighina F. Modulatory effects of $1 \mathrm{~Hz}$ rTMS over the cerebellum on motor cortex excitability. Exp Brain Res. 2007;176(3):440-7.

37. Oulad Ben Taib N, Manto M, Laute MA, Brotchi J. The cerebellum modulates rodent cortical motor output after repetitive somatosensory stimulation. Neurosurgery. 2005;56(4):811-20.

38. Oulad Ben Taib N, Manto M. Trains of transcranial direct current stimulation antagonize motor cortex hypoexcitability induced by acute hemicerebellectomy. J Neurosurg. 2009;111(4):796-806.

39. Heaney CJ, Campeau NG, Lindell EP. MR imaging and diffusion-weighted imaging changes in metronidazole (Flagyl)-induced cerebellar toxicity. AJNR Am J Neuroradiol. 2003;24(8):1615-7.

40. Koeppen $\mathrm{AH}$. The pathogenesis of spinocerebellar ataxia. Cerebellum. 2005;4(1):62-73.

41. Koeppen AH, Michael SC, Knutson MD, Haile DJ, Qian J, Levi S, Santambrogio P, Garrick MD, Lamarche JB. The dentate nucleus in Friedreich's ataxia: the role of iron-responsive proteins. Acta Neuropathol. 2007;114(2):163-73.

42. Storey E, Knight MA, Forrest SM, Gardner RJ. Spinocerebellar ataxia type 20. Cerebellum. 2005;4(1):55-7.

43. Scotti G, Scialfa G, Tampieri D, Landoni L. MR imaging in Fahr disease. J Comput Assist Tomogr. 1985;9(4):790-2.

44. Kanda T, Osawa M, Oba H, Toyoda K, Kotoku J, Haruyama T, Takeshita K, Furui S. High signal intensity in dentate nucleus on unenhanced T1weighted MR images: association with linear versus macrocyclic gadolinium chelate administration. Radiology. 2015;275(3):803-9.

45. Benussi A, Koch G, Cotelli M, Padovani A, Borroni B. Cerebellar transcranial direct current stimulation in patients with ataxia: a double-blind, randomized, sham-controlled study. Mov Disord. 2015;30(12):1701-5.

46. van Rootselaar AF, van der Salm SM, Bour LJ, Edwards MJ, Brown P, Aronica E, Rozemuller-Kwakkel JM, Koehler PJ, Koelman JH, Rothwell JC, Tijssen MA. Decreased cortical inhibition and yet cerebellar pathology in 'familial cortical myoclonic tremor with epilepsy'. Mov Disord. 2007;22(16):2378-85

47. Ganos C, Kassavetis P, Erro R, Edwards MJ, Rothwell J, Bhatia KP. The role of the cerebellum in the pathogenesis of cortical myoclonus. Mov Disord. 2014:29(4):437-43.

48. Luft AR, Kaelin-Lang A, Hauser TK, Buitrago MM, Thakor NV, Hanley DF, Cohen LG. Modulation of rodent cortical motor excitability by somatosensory input. Exp Brain Res. 2002;142(4):562-9.

49. Manto M, Oulad Ben Taib N, Luft AR. Modulation of excitability as an early change leading to structural adaptation in the motor cortex. J Neurosci Res. 2006;83(2):177-80.

50. Quartarone A, Lang N, Rizzo V, Bagnato S, Morgante F, Sant'angelo A, Crupi D, Battaglia F, Messina C, Girlanda P. Motor cortex abnormalities in amyotrophic lateral sclerosis with transcranial direct-current stimulation. Muscle Nerve. 2007;35(5):620-4.

51. Ugawa Y, Genba-Shimizu K, Rothwell JC, Iwata M, Kanazawa I. Suppression of motor cortical excitability by electrical stimulation over the cerebellum in ataxia. Ann Neurol. 1994;36(1):90-6.

52. Dubbioso R, Pellegrino G, Antenora A, De Michele G, Filla A, Santoro L, Manganelli $F$. The effect of cerebellar degeneration on human sensori-motor plasticity. Brain Stimul. 2015;8(6):1144-5. 